\title{
Advances in the Treatment of Advanced Non-Small Cell Lung Cancer
}

\author{
Xianshan Chen ${ }^{1}$, Juncheng Guo ${ }^{2 *}$, Min Guo ${ }^{1}$ \\ ${ }^{1}$ Hainan General Hospital, Haikou, China \\ ${ }^{2}$ Central South University Xiangya School of Medical Affiliated Haikou Hospital, Haikou, China \\ Email: *g2002m@163.com
}

How to cite this paper: Chen, X.S., Guo, J.C. and Guo, M. (2020) Advances in the Treatment of Advanced Non-Small Cell Lung Cancer. Advances in Lung Cancer, 9, 30-40. https://doi.org/10.4236/alc.2020.92004

Received: May 31, 2020

Accepted: June 26, 2020

Published: June 29, 2020

Copyright (c) 2020 by author(s) and Scientific Research Publishing Inc. This work is licensed under the Creative Commons Attribution International License (CC BY 4.0).

http://creativecommons.org/licenses/by/4.0/

\begin{abstract}
In recent years, the incidence of lung adenocarcinoma has been increasing, and now it has become the largest type of non-small cell lung cancer (NSCLC). Currently, treatment of advanced NSCLC consists of several modalities: systemic chemotherapy, local radiation therapy, and targeted therapy (including most recently immunotherapy). In the past decade, the discovery of new molecular subtypes, the search for tumor driver gene mutations, the development of targeted molecular targeted drugs, or targeted therapy to suppress tumor angiogenesis and regulate tumor immune response have been the main directions of NSCLC research and clinical diagnosis and treatment. At present, platinum-based chemotherapy is widely used in NSCLC patients clinically. Platinum-based chemotherapy drugs can effectively prolong the survival time of patients and improve their quality of life, but the incidence of adverse reactions is still high. Therefore, it is necessary to find a drug that can improve the efficacy of patients and reduce the adverse reactions of platinum chemotherapy drugs to NSCLC patients.
\end{abstract}

\section{Keywords}

Non-Small Cell Lung Cancer, Drugs, Targeted, Chemotherapy

\section{Introduction}

The gene mutation status of epidermal growth factor receptor (EGFR) in tumor tissues of non-small-cell lung cancer (NSCLC) is closely related to the efficacy of the epidermal growth factor receptor tyrosine kinase inhibitors (egfr-tkis). However, in some cases, insufficient tumor tissue, too few tumor cell components, or difficulty in obtaining tumor tissue can result in the inability to detect tisses-dependent mutations in the EGFR gene. Currently, the use of plasma or serum for EGFR gene testing has not been approved by current guidelines or is not widely used in 
clinical practice to guide the treatment of egfr-tki [1]. Studies have shown that smoking is the main risk factor of lung cancer, and about $85 \%-90 \%$ of lung cancer is caused by smoking, including active smoking and passive smoking. Therefore, prohibition and control of smoking is an effective means to prevent lung cancer [2]. In today's world, with the aggravation of environmental pollution, as well as the change of living rules and dietary structure, the incidence of lung cancer has shown a progressive rising trend, and it has become one of the most common malignant tumor diseases in the world [3]. Lung cancer is a serious threat to human health due to its high degree of malignancy, rapid development and low five-year survival rate. Therefore, the formulation of standardized individualized treatment regimens and accurate evaluation of the curative effect after treatment plays an important role in prolonging the survival period and improving the quality of life. According to histopathological classification, lung cancer can be divided into non-small cell carcinoma and small cell carcinoma [4]. The frequency of EGFR gene mutation in non-small cell lung cancer is related to the clinical background such as race, gender, smoking history and pathological type.

Non-small cell carcinoma includes squamous cell carcinoma, adenocarcinoma and large cell carcinoma, accounting for more than $80 \%$ of lung cancer. The treatment methods of advanced non-small cell lung cancer include surgery, chemotherapy, radiotherapy, targeted therapy, traditional Chinese medicine and immunotherapy [5]. About 75 percent of patients are found to be at an advanced stage and have no chance of surgery. A large number of clinical trials have confirmed that platinum-based chemotherapy is the first-line treatment for advanced non-small cell lung cancer [6], but chemotherapy seems to have entered the plateau stage at present. With the development of various technologies, epidermal growth factor receptor (EGFR), a single transmembrane glycoprotein with a molecular weight of $170 \mathrm{KDa}$, is a receptor of epidermal growth factor-mediated signal transduction and cell proliferation. EGFR is one of the four receptor members of HER family and belongs to tyrosine kinase receptor. Tyrosine kinase consists of two subunits, the $\mathrm{N}$ subunit and the $\mathrm{C}$ subunit. ATP binds to the binding site between the two subunits. When the ligand binds to the EGFR extracellular region, the EGFR molecules form isomeric dimers, and can also bind to other her-family molecules to form heterodimers. The phosphorus ATP is transferred to the regulation of tyrosine residues, tyrosine kinase is activated, activated tyrosine residues phosphorylation, and guide the downstream protein phosphorylation, activate the downstream RAS MAPK, PI3K AKT, STAT kinase pathway, which can lead to proliferation, angiogenesis, metastasis and apoptosis escape occurred related to tumor cell biology characteristics, such as [7] [8], Egfr-tkis has gradually attracted the attention of doctors, and the wide application of egfr-tkis has extended the survival time and improved the quality of life of many lung cancer patients. Currently, egfr-tkis has become the first-line treatment for some patients with positive EGFR gene mutation. Both egfr-tkis and chemotherapy have been widely and mature used in clinical work, and many patients have be- 
nefited from them. However, whether patients can continue to use the original treatment regimen and whether they need to change the treatment regimen needs to be evaluated by medical imaging. Therefore, accurate evaluation also plays an important role in the treatment of patients.

\section{Platinum-Based Chemotherapy}

Platinum-based chemotherapy is the standard first-line treatment for advanced non-small cell lung cancer [6]. The National Cancer Institute of Canada [9] conducted a study on vinblastamide + cisplatin (VP), cyclophosphamide + A Prospective randomized controlled trials of three groups of mycin + cisplatin (CAP) and best supportive treatment (BSC) showed that the objective response rates of the CAP group and the VP group were $15.3 \%$ and $25.3 \%$, respectively. The median survival time was 32.6 weeks, 24.7 weeks, and 17 weeks, respectively. The median survival time of the chemotherapy group was significantly longer than that of the best supportive treatment group, but the chemotherapy side effects of the chemotherapy group were significantly increased, including the incidence of leukopenia in the CAP and VP groups. The rates were $37.8 \%$ and $40.0 \%$, respectively, and the incidence of severe vomiting in the CAP and VP groups was $12.2 \%$ and $23.3 \%$, respectively. The platinum-based two-drug combination scheme is the current first-line chemotherapy for non-small cell lung cancer, such as paclitaxel combined with platinum, cytidine combined with platinum, and vinblastine combined with platinum. The results of clinical trials have shown that [10] [11] the two-drug combination chemotherapy is superior to the single-drug chemotherapy in terms of treatment efficacy and survival time. However, the therapeutic effect of the three-drug combination regimen did not show better efficacy than the two-drug combination regimen, which could not significantly prolong the survival period, and increased the side effects of chemotherapy and the associated risk of death.

\section{Application of Pemetrexed Disodium in the Treatment of Advanced Non-Small Cell Lung Cancer}

\subsection{Mechanism of Action of Pemetrexed Disodium}

In recent years, pemetrexed has played an important role in the first-line, secondline and maintenance treatment of lung cancer. Pemetrexide is an anti-folic acid preparation containing pyrropyrimidine groups, which can inhibit the activities of thymidylate synthase, glycamine nucleotide formyl transferase and dihydrofolate reductase, which are all essential enzymes for the synthesis of folic acid [12]. Pemetrexed inhibits DNA synthesis of tumor cells by inhibiting folic acid production, thus inhibiting tumor growth. It is a new multi-target anti-metabolic drug.

\subsection{Second-Line Treatment of Pemetrexed Disodium in Advanced Non-Small Cell Lung Cancer}

Hanna et al. [13] conducted a large randomized, controlled phase III clinical trial, randomly dividing 571 patients with NSCLC who had progressed after plati- 
num-based chemotherapy into the docetaxel group and pemetrexed The results of the group showed that the overall remission rates were $9.1 \%$ and $8.8 \%$, the median PFS was 2.9 months, and the median OS was 8.3 and 7.9 months, respectively. There was no statistical difference between the two groups. However, compared with the docetaxel group, the pemetrexed group can reduce the significant side effects of chemotherapy, so pemetrexed can be used as an option in the second-line treatment of advanced NSCLC.

\subsection{First-Line Treatment of Pemetrexed Disodium in Advanced NSCLC}

People began to explore the application of pemetrexed in the second-line treatment of advanced NSCLC after it was gradually confirmed in clinical practice that pemetrexed has an accurate curative effect and low toxic and side effects. Scagliotti [14] and others conducted a III period of large, randomized, controlled clinical trial, compared the pemetrexed disodium combined cisplatin and gemcitabine combined cisplatin for advanced NSCLC first-line treatment the curative effect and adverse reactions. The results showed that there was no significant statistical difference in the efficacy between the two groups. The median OS of pemetrexed disodium combined with cisplatin and gemcitabine combined with cisplatin were both 10.3 months, and the median PFS were 4.8 months and 5.1 months, respectively. However, the occurrence of adverse events such as blood system toxicity in pemetrexide combined with cisplatin was lower than that in gemcitabine combined with cisplatin group, and there was a statistical difference between the two groups. At the same time, subgroup analysis showed that compared with the two groups, pemetrexed combined with cisplatin had longer median OS in large cell carcinoma and adenocarcinoma. The median OS in large cell carcinoma was 10.4 months and 6.7 months, and the median OS in adenocarcinoma was 12.6 months and 10.9 months, respectively. For squamous cell carcinoma, the median OS was 9.4 months and 10.8 months, respectively. A about pemetrexed combined cisplatin with changchun marina combined cisplatin in advanced non-small cell lung cancer first-line treatment of contrast research also indicated that there was no significant difference between treatment effect between two groups, all can effectively prolong patient survival time, but the side effects of pemetrexed is less, can be used as advanced non-small cell lung cancer using first-line treatment promotion.

\subsection{Maintenance Therapy of Pemetrexed in Advanced Non-Small Cell Lung Cancer}

Maintenance therapy can be divided into continuous maintenance therapy and dressing change maintenance therapy. Continuation of maintenance therapy refers to the use of a drug used in first-line therapy after 4 to 6 cycles of first-line medication, if there is no disease progression. Replacement maintenance therapy refers to the use of another drug not included in the first-line treatment regimen after 4 to 6 cycles of first-line treatment if no disease progression occurs. Ci- 
uleanu et al. [15] conducted a phase III clinical study on maintenance therapy of pemetrex in non-small cell lung cancer (JMEN). The results showed that the median survival (OS) of the pemetrexide maintenance group and the placebo group were 13.4 months and 10.6 months, respectively $(\mathrm{p}=0.012)$, with statistically significant differences between the two groups. It should be noted that the survival benefit was greater in non-squamous cell carcinoma patients (15.5 months vs 10.3 months), while there was no statistically significant difference between the two groups in squamous cell carcinoma patients. Therefore, the US food and drug administration [FDA] approved pemetrex for maintenance therapy in patients with platinum-based non-squamous cell non-small cell lung cancer with no progression of disease, and it is recommended in the NCCN guidelines.

\section{Targeted Drug Therapy for Advanced Non-Small Cell Lung Cancer}

\subsection{EGFR TKIs}

EGFR is a transmembrane glycoprotein widely distributed in the cell membrane of human body, and is the receptor of epithelial growth factor cell proliferation and signal transduction. High or abnormal expression of EGFR exists in many solid malignant tumors, which leads to the enhancement of downstream signal transduction, which leads to the proliferation, angiogenesis, tumor invasion and metastasis of tumor cells and inhibits the apoptosis of tumor cells. Egfr-tkis (epidermal growth factor receptors, tyrosine kinase inhibitors) bind to the atp-binding site in the EGFR tyrosine kinase region, blocking signaling, inhibiting tumor cell proliferation, and promoting apoptosis. After the introduction of egfr-tkis, the quality of life of patients has been improved and the survival time of patients has been extended, which has attracted the attention of doctors and patients. At present, the clinical application of the most is the first generation of egfr-tkis, representative of erlotinib, gefitinib and ectinib.

\subsubsection{Second-Line Treatment of Egfr-Tkis in Advanced Non-Small Cell Lung Cancer}

Erlotinib was approved by the US FDA in November 2014 for the treatment of non-small cell lung cancer. The TRUST trial is a large clinical study offering tarcay in patients with advanced NSCLC. TRUST [16] the primary purpose is to receive at least one standard chemotherapy (III B/IV) late NSCLC patients with tarceva therapy. This study demonstrates, on a large scale, the superior therapeutic benefit in the treatment of second- and third-line non-small cell lung cancer in tarceva. Ge, Chen [17] is a large randomized, controlled, double-blind clinical trial comparing the efficacy of erlotinib and placebo in second-line or third-line treatment of non-small cell lung cancer. The median PFS of erlotinib group and placebo group was 2.2 months and 1.8 months, respectively, $\mathrm{p}<0.001$, indicating a clinically significant difference. Median overall survival was 6.7 months and 4.7 months, respectively, $\mathrm{p}<0.001$. The difference between the two groups was clinically significant. Therefore, erlotinib can be used as a second-line or 
third-line treatment for advanced non-small cell lung cancer. Ge, Chen [18] and others conducted a in the treatment of patients with advanced NSCLC second-line treatment were retrospectively analyzed, the results show that the second line therapy after the treatment applied median survival time was 14.4 months, the objective response rate was $24.6 \%$, it is worth mentioning that in female, adenocarcinoma, nonsmoking patients, showed better survival benefit.

\subsubsection{First-Line Treatment of Egfr-Tkis in Advanced Non-Small Cell Lung Cancer}

The above studies have clarified the efficacy and safety of egfr-tkis in second-line treatment of advanced NSCLC, so people began to study the efficacy and safety of egfr-tkis in first-line treatment. The IPASS study [19] is a landmark of targeted therapy for lung cancer. It is an open, randomized, multicenter phase III clinical study evaluating the efficacy, safety, and tolerability of gefitinib versus paclitaxel/carboplatin two-drug chemotherapy in first-line treatment of advanced Asian (stage IIIB/IV) NSCLC. The results showed that for patients with EGFR mutations, the median PFS of the gefitinib group was significantly better than that of the chemotherapy group (9.5 months vs 6.3 months), and for patients without EGFR mutations, the median PFS of the chemotherapy group was significantly better than that of the gefitinib group (5.5 months vs 1.5 months). The IPASS study identified the important role and role of EGFR mutations in the clinical selection of egfr-tkis. OPTIMAL research [20] is a multicenter, randomized, open compare it for two medicine and platinum-based chemotherapy first-line treatment of EGFR mutation positive patients with NSCLC, III period clinical research. OPTIMAL research into the group of 165 cases of EGFR mutation positive III B/IV NSCLC patients, according to the 1:1 random grouping, a set of given it for $150 \mathrm{mg} / \mathrm{d}$, another group to give 4 cycles of gemcitabine plus carboplatin chemotherapy. The results showed that erlotinib could prolong the median PFS by 2 times compared with the chemotherapy group (13.1 months vs 4.6 months, $\mathrm{HR}=0.16, \mathrm{p}<0.0001$ ), and the adverse reactions in the chemotherapy group were more common than those in the erlotinib group.

\subsection{Application of Anti-Vegf Monoclonal Antibody in Advanced Non-Small Cell Lung Cancer}

The growth and survival of tumor cells depend on blood vessels to provide oxygen and nutrients. Vascular endothelial growth factor (VEGF) can promote cell division, proliferation and migration, and plays an important role in tumor angiogenesis. Bevacizumab is an anti-vegf monoclonal antibody that has been shown to inhibit tumor growth. Sandler [21] research is a study of III/IV non scale random III stage non-small cell lung cancer clinical trial, 878 patients were involved in the clinical trials, randomized to paclitaxel/carboplatin chemotherapy group and paclitaxel alone/carboplatin in combination with bevacizumab group, the results show that the beacizumab bead sheet resistance compared with the pure chemotherapy group median PFS (6.2 vs. 4.5 months, $\mathrm{p}<0.001)$ and OS $(12.3$ 
months vs $10.3, \mathrm{p}=0.003)$ are extended, and the differences were statistically significant. The results showed that bevacizumab combined with chemotherapy could prolong the survival of patients with non-squamous non-small cell lung cancer. However, the treatment-related mortality increased in the bevacizumab combined with chemotherapy group ( 5 cases died from pulmonary hemorrhage, 5 cases died from leukopenia, 2 cases died from gastrointestinal bleeding, and 3 cases died from cerebrovascular disease), but no adverse events were observed, and the safety was within the tolerance range. AVAiL study [22] enrolled 1043 initially treated patients with advanced non-small cell lung cancer, and the results showed that the gemcitabine/cisplatin combined with bevacizumab group had longer PFS and OS than the chemotherapy group alone. The results showed that the efficacy of bevacizumab was similar between the two groups with different dosages ( $15 \mathrm{mg} / \mathrm{kg}$ in the high-dose group and $7.5 \mathrm{mg} / \mathrm{kg}$ in the low-dose group). With the confirmation of the efficacy and safety of bevacizumab combined with multiple chemotherapy regimens in the first-line treatment of advanced non-small cell carcinoma, the clinical benefits of bevacizumab combined with egfr-tkis have been investigated. The results of the Takashi Seto [23] trial showed that for patients with positive EGFR mutation, the progression-free survival time after erlotinib combined with bevacizumab was increased by 6.3 months compared with erlotinib alone $(\mathrm{p}=0.0015 ; \mathrm{HR}=0.54)$, and the difference between the two was statistically significant. At the same time, no new adverse events occurred after chemotherapy combined with bevacizumab, which provided a basis for the combined treatment of TKI and anti-angiogenesis drugs.

\section{Important Application of PET/CT in Lung Cancer}

After receiving treatment, patients with advanced non-small cell lung cancer need to use medical imaging to evaluate the changes in the lesions, so as to determine the efficacy of the treatment regimen and whether to change the treatment regimen. Therefore, accurate evaluation also plays an important role in the treatment of advanced non-small cell lung cancer. At present, the reciist version 1.0 issued in 2000 and RECIST version 1.1 revised in 2009 are commonly used in clinical practice. With the rapid development of medical imaging, PET/CT came into being. PET/CT was used to evaluate the efficacy from the functional and metabolic levels of lesions. In 1999, the European organization for research and treatment of cancer (EORTC) published a standard for evaluating the efficacy of tumor therapy based on the standardized intake of 18f-fdg (18f-deoxyglucose) PET (SUV value) [24].

\subsection{The Application of PET/CT in the Diagnosis of Lung Cancer}

Currently X-ray sternum, computer tomography (computed tomography, CT) scanning imaging for diagnosis of lung cancer is the most common method, can help clinicians to make a preliminary diagnosis and staging, determined that most lung cancer and then application of lung puncture or fiber bronchoscope for organization pathology inspection to diagnosis, but for some individual is 
difficult to take the lungs pathology of solitary nodule diagnosis has certain restrictions. PET/CT is used to describe and compare the uptake level of $18 \mathrm{f}$-fdg in tissues according to the standard intake value (SUV), so as to identify the benign and malignant lesions, so as to achieve the purpose of early diagnosis, accurate staging and reasonable treatment. Duhaylongsod et al. [25] summarized the PET/CT results of 53 patients with single solitary pulmonary nodules, and found that the standard uptake ratio of malignant lesions (SUV) was $5.9 \pm 2.7$, while that of benign lesions was $2.0 \pm 1.7$, and the difference between the two was statistically significant. If the value of SUV $\geq 2.5$ is used as the diagnostic criteria for lung cancer, the sensitivity of PET/CT to the diagnosis of lung cancer is $100 \%$ and the specificity is $79 \%$. The false positive rate of PET/CT in the diagnosis of lung cancer is $10 \%$, mainly due to the increased glycolysis of inflammatory granulomatous diseases (such as tuberculosis, pulmonary sarcoidosis, pulmonary aspergillosis, etc.), and the activation of hexose phosphate bypass phagocytosis, which is higher than the basal value, leading to increased metabolism. The false negative rate of PET/CT was less than $5 \%$, and the main reason for false negative was that the volume effect could be caused when the lesion length diameter was less than $7 \mathrm{~mm}$, and the low glucose transporter protein in the adenocarcinoma, partial bronchiolar alveolar carcinoma and carcinoid carcinoma with high differentiation degree was caused. Lardinois et al. [26] also reported that in 40 patients with lung cancer, the diagnostic accuracy of PET/CT was $98 \%$.

\subsection{The Application of PET/CT in Lung Cancer Staging}

TNM staging of lung cancer is an important step in the diagnosis of lung cancer and an important basis for the selection of treatment options and prognosis assessment. Multiple practices have shown that TNM stage is highly correlated with the prognosis of lung cancer patients [27] [28]. CT can evaluate tumor size and its relationship with surrounding tissues from the perspective of anatomical structure, while PET can evaluate the tumor from the perspective of molecular metabolism. PET/CT can better reflect the tumor size and its relationship with surrounding tissues, so as to distinguish tumor from atresia, tumor from obstructive pneumonia, etc. Current imaging techniques (CT, MRI, etc.) are not clear in the diagnosis of lymph node metastasis of lung cancer. However, PET/CT relies on the biochemical metabolism of the lesion site to display the lesion, which belongs to functional imaging. Konishi et al. [29] analyzed 306 lymph nodes of 54 patients, and the results showed that the sensitivity of PET/CT and CT were 73\% and $97 \%$, respectively, and the specificity was $98 \%$ and $96 \%$. Liu lanping et al. [30] compared the postoperative pathological examination results of 68 NSCLC patients who underwent radical surgery with the preoperative CT and PET/CT results, and the results showed that the accuracy of CT and PET/CT in diagnosing $\mathrm{N}$ staging was $54.4 \%$ and $76.5 \%$, respectively, with statistically significant differences $(p<0.05)$. It indicates that PET/CT has a higher clinical value than $\mathrm{CT}$ in the diagnosis of mediastinal lymph node metastasis of NSCLC and can more accurately determine the $\mathrm{N}$ stage. 


\section{Conclusion}

With the continuous progress of science and technology, lung cancer treatment is also progressing, lung cancer patients' five-year survival rate and quality of life has been improved. Medical imaging is needed to evaluate the changes of lesions after the treatment of patients, so as to determine whether the treatment plan needs to be changed. Therefore, accurate evaluation plays an important role in the treatment of patients with lung cancer. Since the advent of PET/CT, it has played an important role in the diagnosis, staging, post-treatment staging and efficacy evaluation of lung cancer. Of course, PET/CT also has some shortcomings, such as high cost and long time of examination. With the development of medical imaging technology, these shortcomings will be gradually improved and widely used in clinical practice, playing an important role in the diagnosis and treatment of lung cancer patients.

\section{Funding}

Funded by the Scientific Research Project of Health and Family Planning Industry of Hainan Province, project number: 18A200050.

\section{Conflicts of Interest}

The authors declare no conflicts of interest regarding the publication of this paper.

\section{References}

[1] Siegel, R., Ma, J., Zou, Z., et al. (2014) Cancer Statistics. CA: A Cancer Journal for Clinicians, 64, 9-29. https://doi.org/10.3322/caac.21208

[2] Govindan, R., Page, N., Morgensztern, D., et al. (2006) Changing Epidemiology of Small-Cell Lung Cancer in the United States over the Last 30 Years: Analysis of the Surveillance, Epidemiologic and End Results Database. Journal of Clinical Oncology, 24, 4539-4544. https://doi.org/10.1200/JCO.2005.04.4859

[3] Chen, Q.F., Luo, J., Wu, C., Lu, H.S., Cai, S.Q., Bao, C.X., Liu, D.M. and Kong, J.L. (2020) The miRNA-149-5p/MyD88 Axis Is Responsible for Ursolic Acid-Mediated Attenuation of the Stemness and Chemoresistance of Non-Small Cell Lung Cancer Cells. Environmental Toxicology, 35, 561-569. https://doi.org/10.1002/tox.22891

[4] Herbst, R.S., Heymach, J.V. and Lippman, S.M. (2008) Molecular Origins of Cancer: Lung Cancer. The New England Journal of Medicine, 359, 1367-1380. https://doi.org/10.1056/NEJMra0802714

[5] Alberg, A.J., Ford, J.G., Samet, J.M., et al. (2007) Epidemiology of Lung Cancer. ACCP Evidence-Based Clinical Practice Guidelines (2nd Edition). Chest, 132, 29S-55S. https://doi.org/10.1378/chest.07-1347

[6] Finazzi, T., Ronden-Kianoush, M.I., Spoelstra, F.O.B., et al. (2020) Stereotactic Ablative Radiotherapy in Patients with Early-Stage Non-Small Cell Lung Cancer and Co-Existing Interstitial Lung Disease. Acta Oncologica (Stockholm, Sweden), 59, 569-573. https://doi.org/10.1080/0284186X.2020.1730002

[7] Wang, C.D., Qiao, W.L., Jiang, Y.T., Zhu, M., Shao, J., Wang, T., Liu, D. and Li, W.M. (2020) The Landscape of Immune Checkpoint Inhibitor plus Chemotherapy 
versus Immunotherapy for Advanced Non-Small-Cell Lung Cancer: A Systematic Review and Meta-Analysis. Journal of Cellular Physiology, 235, 4913-4927. https://doi.org/10.1002/jcp.29371

[8] Oyedele, A.S., Bogan, D.N. and Okoro, C.O. (2020) Synthesis, Biological Evaluation and Virtual Screening of Some Acridone Derivatives as Potential Anticancer Agents. Bioorganic \& Medicinal Chemistry, 28, Article ID: 115426. https://doi.org/10.1016/j.bmc.2020.115426

[9] Boff, L., Persich, L., Brambila, P., Ottoni, F.M., et al. (2020) Investigation of the Cytotoxic Activity of Two Novel Digitoxigenin Analogues on H460 Lung Cancer Cells. Anti-Cancer Drugs, 31, 452-462. https://doi.org/10.1097/CAD.0000000000000872

[10] Rapp, E., Pater, J.L., Willan, A., et al. (1988) Chemotherapy Can Prolong Survival in Patients with Advanced Non-Small-Cell Lung Cancer-Report of a Canadian Multicenter Randomized Trial. Journal of Clinical Oncology, 6, 633-641. https://doi.org/10.1200/JCO.1988.6.4.633

[11] Delbaldo, C., Michiels, S., Syz, N., Soria, J.C., Le Chevalier, T. and Pignon, J.P. (2004) Benefits of Adding a Drug to a Single-Agent or a 2-Agent Chemotherapy Regimen in Advanced Non-Small Cell Lung Cancer: A Met-Analysis. JAMA, 292, 470-484. https://doi.org/10.1001/jama.292.4.470

[12] Guo, T.S., Huang, J.M., Wan, X.Y., Chen, P.X., Du, S.S. and Chen, Q.P. (2020) lncRNA Metastasis-Associated Lung Adenocarcinoma Transcript 1 Promotes Proliferation and Invasion of Non-Small Cell Lung Cancer Cells via Down-Regulating miR-202 Expression. Cell Journal, 22, 375-385.

[13] Hanna, N., Shepherd, F.A., Fossella, F.V., et al. (2004) Randomized Phase III Trial of Pemetrexed versus Docetaxel in Patients with Non-Small Cell Lung Cancer Previously Treated with Chemotherapy. Journal of Clinical Oncology, 22, 1589-1597. https://doi.org/10.1200/JCO.2004.08.163

[14] Scagliotti, G.V., Parikh, P., von, P.J., et al. (2008) Phase III Study Comparing Cisplatin plus Gemcitabine with Cisplatin plus Pemetrexed in Chemotherapy-Naive Patients with Advanced-Stage Non-Small Cell Lung Cancer. Journal of Clinical Oncology, 26, 3543-3551. https://doi.org/10.1200/JCO.2007.15.0375

[15] Cluleanu, T.E., Brodowicz, T., Belani, C.P., et al. (2009) Maintenance Pemetrexed plus Best Supportive Care (SBC) versus Placebo plus SBC: A Randomized III Study. The Lancet, 3749, 1432-1440. https://doi.org/10.1016/S0140-6736(09)61497-5

[16] Marcello, T., Cesare, G., Stefano, C., et al. (2009) An Expanded Access Program of Erlotinib (Tarceva) in Patients with Advanced Non-Small Cell Lung Cancer (NSCLC): Data Report from Italy. Lung Cancer, 64, 199-206.

https://doi.org/10.1016/j.lungcan.2008.09.001

[17] Chen, G., Zhang, Y.Z., Deng, H.W., Tang, Z.L., Mao, J.J. and Wang, L. (2020) Pursuing for the Better Lung Cancer Therapy Effect: Comparison of Two Different Kinds of Hyaluronic Acid and Nitroimidazole Co-Decorated Nanomedicines. Biomedicine \& Pharmacotherapy, 125, Article ID: 109988. https://doi.org/10.1016/j.biopha.2020.109988

[18] Song, Y.X., Zhou, B., Du, X.Y., Wang, Y., Zhang, J., Ai, Y.Q., Xia, Z.J. and Zhao, G.F. (2020) Folic Acid (FA)-Conjugated Mesoporous Silica Nanoparticles Combined with MRP-1 siRNA Improves the Suppressive Effects of Myricetin on Non-Small Cell Lung Cancer (NSCLC). Biomedicine \& Pharmacotherapy, 125, Article ID: 109561. https://doi.org/10.1016/j.biopha.2019.109561 
[19] Mok, T. and Wu, Y.L. (2008) Phase III, Randomised Open-Label, First-Line Study of Gefetinib versus Carboplatin/Paclitacel in Clinically Selected Patients with Advanced Non-Small Lung Cancer. Annals of Oncology, 19, Abstract LBA2.

[20] Zhou, C., Wu, Y.L. and Chen, G. (2011) Erlotinib versus Chemotherapy as First-Line Treatment for Patients with Advanced EGFR Mutation-Positive Non-Small-Cell Lung Cancer (Optimal, CTONG-0802): A Multicentre, Open-Label, Randomised, Phase 3 Study. The Lancet Oncology, 12, 735-742. https://doi.org/10.1016/S1470-2045(11)70184-X

[21] Sandler, A., Gray, R., Perry, M.C., et al. (2006) Paclitacel-Carboplatin Alone or with Bevacizumab for Non-Small Cell Lung Cancer. The New England Journal of Medicine, 355, 2542-2550. https://doi.org/10.1056/NEJMoa061884

[22] Reck, M., von Pawel, J., Zatloukal, P., et al. (2009) Phase III Trial of Cisplatin-Plus Gemcitabine with either Placebo or Bevacizumab as First-Line Therapy for Nonsquamous Non-Small-Cell Lung Cancer: AVAil. Journal of Clinical Oncology, 27, 1227-1234. https://doi.org/10.1200/JCO.2007.14.5466

[23] Seto, T., Kato, T., et al. (2014) Erlotinib Alone or with Bevacizumab as First-Line Therapy in Patients with Advanced Non-Squamous Non-Small Cell Lung Cancer Harbouring EGFR Mutations (JO25567): An Open-Label, Randomised, Multicentre, Phase 2 Study. The Lancet Oncology, 15, 1236-1244.

https://doi.org/10.1016/S1470-2045(14)70381-X

[24] Young, H., Baum, R., Cremerius, U., et al. (1999) Measurement of Clinical and Subclinical Tumor Response Using [18F]-Fluorodeoxyglucose and Positron Emission Tomography: Review and 1999 EORTC Recommendations. European Organization for Research and Treatment of Cancer (EORT C) PET Study Group. European Journal of Cancer, 35, 1773-1782. https://doi.org/10.1016/S0959-8049(99)00229-4

[25] Duhaylongsod, F.G., Lowe, V.J., Patz, E.F., et al. (1995) Detection of Primary and Recurrent Lung Cancer by Means of 18F-FDG PET. Journal of Thoracic and Cardiovascular Surgery, 110, 130-139. https://doi.org/10.1016/S0022-5223(05)80018-2

[26] Lardinois, D., Weder, W., Hany, T.F., et al. (2003) Staging of Non Small Cell Lung Cancer with Integrated Positron Emission Tomography and Computed Tomography. The New England Journal of Medicine, 348, 2500-2507. https://doi.org/10.1056/NEJMoa022136

[27] Hany, T.F., Steinert, H.C., Goerres, G.W., et al. (2002) PET Diagnostic Accuracy: Improvement with in Line PET/CT System: Initial Results. Radiology, 225, 575-581. https://doi.org/10.1148/radiol.2252011568

[28] Qin, X.S. and Tian, M.X. (2006) Prognosis of Lung Cancer. Journal of Practical Medicine, 13, 3310-3311.

[29] Konishi, J., Yamazaki, K., Tsukamoto, E., et al. (2003) Mediastinal Lymph Node Staging by FDG PET in Patients with Non Small Cell Lung Cancer: Analysis of False Positive FDG PET Findings. Respiration, 70, 500-506.

https://doi.org/10.1159/000074207

[30] Liu, L.P., Yu, J.M., Guo, H.B., et al. (2007) Application of 18f-fdg PET/CT Imaging in Lymph Node Metastasis and Staging of Non-Small Cell Lung Cancer. Chinese Journal of Nuclear Medicine and Molecular Imaging, 27, 264-266. 